\title{
Ensino da prática baseada em evidências: perspectivas de enfermeiros gestores universitários
}

\section{Teaching evidence-based practice: perspectives of university nurse managers}

\section{La enseñanza de la práctica basada en evidencias: perspectivas de enfermeros gestores universitarios}

Recebido: 05/03/2020

Aprovado: 22/08/2020

Publicado: 14/04/2021
Fernanda Carolina Camargo ${ }^{1}$ Gilberto de Araújo Pereira ${ }^{2}$ Luan Augusto Alves Garcia ${ }^{3}$ Marija Antunes ${ }^{4}$ Adriana Oliveira de Freitas Araujo 5 Álvaro da Silva Santos 6

0 presente estudo tem como objetivo compreender o ensino da prática baseada em evidências na perspectiva de enfermeiros gestores universitários. Trata-se de estudo por métodos mistos, conforme concepção da Prática Baseada em Evidências, e pela Teoria de Difusão de Inovações, conduzido por entrevistas individuais semiestruturadas, por abordagem hermenêutico-dialética, junto à amostra intencional de 10 enfermeiros gestores de uma universidade pública. 0 ensino da prática baseada em evidências se dava por iniciativas pontuais de docentes, durante estágios por levantamento de uma questão clínica e busca de evidências para respondê-la, ou em atividades de busca e análise crítica de artigos científicos. Os resultados apoiam a orientação da matriz educacional na formação de enfermeiros e indicam que aspectos contextuais influenciadores da adoção da prática baseada em evidências no ensino foram reconhecidos, recomendando incrementar tanto o ensino, como a produção de conhecimento sobre a temática.

Descritores: Enfermagem baseada em evidências; Prática clínica baseada em evidências; Pesquisa em enfermagem; Docentes de enfermagem; Educação superior.

This study aims to understand the teaching of evidence-based practice from the perspective of university nurse managers. It is a study by mixed methods, according to the conception of Evidence-Based Practice, and by the Theory of Diffusion of Innovations, conducted by semi-structured individual interviews, using a hermeneutic-dialectic approach with the intentional sample of 10 nurse managers from a public university. The teaching of evidence-based practice took place through specific initiatives by teachers, during internships to raise a clinical question and search for evidence to answer it, or in search and critical analysis of scientific articles. The results support the orientation of the educational matrix in the training of nurses and indicate that contextual aspects influencing the adoption of evidence-based practice in teaching were recognized, recommending to increase both teaching and the production of knowledge on the subject.

Descriptors: Evidence-based nursing; Evidence-based practice; Nursing research; Faculty, Nursing; Education, Higher.

Este estudio tiene como objetivo comprender la enseñanza de la práctica basada en evidencias desde la perspectiva de enfermeros gestores universitarios. Se trata de un estudio de métodos mixtos, según el concepto de la Práctica Basada en evidencias y la Teoría de la Difusión de Innovaciones, realizado por medio de entrevistas individuales semiestructuradas, utilizando un enfoque hermenéutico-dialéctico, con una muestra intencional de 10 enfermeros gestores de una universidad pública. La enseñanza de la práctica basada en evidencias se produjo a través de iniciativas específicas de los profesores, durante las fases de análisis de una pregunta clínica y búsqueda de evidencia para responderla, o en actividades de búsqueda y análisis crítico de artículos científicos. Los resultados apoyan la orientación de la matriz educativa en la formación de los enfermeros e indican que los aspectos contextuales que influyen en la adopción de la práctica basada en las evidencias en la enseñanza fueron reconocidos, recomendando incrementar tanto la enseñanza, como la producción de conocimiento sobre el tema.

Descriptores: Enfermería basada en la evidencia; Práctica clínica basada en la evidencia; Investigación en enfermería; Docentes de enfermería; Educación superior.

1. Enfermeira. Especialista em Saúde da Família. Mestre e Doutora em Atenção à Saúde. Chefe do Setor Vigilância e Segurança do Paciente do Hospital de Clínicas da Universidade Federal do Triângulo Mineiro (UFTM), Uberaba, MG, Brasil. ORCID: 0000-0002-1048-960X E-mail: fernandaccamargo@yahoo.com.br

2. Estatístico. Mestre e Doutor em Estatística. Professor Adjunto do curso de graduação em Enfermagem da UFTM, Uberaba, MG, 0RCID: 0000-0002-9149-6368

E-mail: pereira_gilberto@yahoo.com.br

3. Enfermeiro. Especialista em Gestão Pública. Mestre em Atenção à Saúde. Doutorando em Atenção à Saúde pela UFTM, Uberaba, MG, Brasil. ORCID: 0000-0003-0984-2688 E-mail: luangarciatpc@yahoo.com.br

4. Enfermeira. Uberaba, MG, Brasil. ORCID: 0000-0003-2281-3304 E-mail: marija.enf@gmail.com

5. Enfermeira. Especialista em Saúde do Adulto na modalidade Residência Multiprofissional em Saúde, Uberaba, MG, Brasil. ORCID: 0000-0002-4863-1667 E-mail: adriannafreitas_@hotmail.com

6. Enfermeiro. Mestre em Administração em Serviços de Saúde. Doutor em Ciências Sociais. Pós Doutor em Serviço Social. Professor Associado do curso de graduação em Enfermagem e nos Programas de Pós-Graduação em Atenção à Saúde e Psicologia da UFTM, Uberaba, MG, Brasil. ORCID 0000-0002-8698-5650 E-mail: alvaroenf@hotmail.com 


\section{INTRODUÇÃO}

A

s mudanças sociais globais implicam sobre os sistemas de valores da Enfermagem enquanto profissão, exigindo a adaptação de suas competências. Novos conhecimentos são exigidos para a prática da Enfermagem em uma sociedade dinâmica e mutável ${ }^{1}$. Na contemporaneidade, observa-se ampla disponibilização de publicações científicas. Entretanto, sua utilização crítica e apropriação no mundo prático ainda apresenta barreiras ${ }^{1,2}$.

De forma geral, a prática baseada em evidências é a competência dada por habilidades específicas para identificação e análise crítica de publicações científicas relevantes com intuito de transformar o conhecimento produzido, através das pesquisas existentes ou da condução de novas pesquisas, em informação segura para a tomada de decisão dos enfermeiros. Visa associar as evidências à expertise profissional e às preferências das pessoas, famílias e comunidades assistidas pelos serviços de saúde ${ }^{2}$.

Mudanças rumo à prática baseada em evidências auxiliam enfermeiros a transpor uma cultura de prestação de cuidados baseada na tradição ou repetição, para uma prática que amplie a qualidade assistencial, a segurança do paciente e o controle dos custos ${ }^{2,3}$. Além disso, exigem dos enfermeiros liderança transformacional para engajamento de equipes e implementação de novas práticas, como também a capacidade de planejar, avaliar e disseminar as mudanças pela incorporação de evidências junto a pessoas assistidas, comunidade científica, e formuladores de políticas. Também é necessário que tenham habilidades para análise da qualidade das evidências disponíveis, a qual requer conhecimentos sobre: diferentes delineamentos de pesquisas, análises estatísticas e métodos para síntese das evidências, como revisões e metaanálise. Acrescenta-se a importância das habilidades em informática para busca das pesquisas, periódicos indexados e bases de dados ${ }^{2,3}$.

Sobremaneira, essa prática ainda é desafiadora à Enfermagem. Uma das principais dificuldades se dá pela competência individual dos enfermeiros para seu desempenho ${ }^{3}$. Sobretudo, educar estudantes de Enfermagem para a prática baseada em evidências é fundamental. Tendo em vista o papel primordial que as universidades têm na revisão e atualização da formação, emerge o questionamento: Como ocorre o ensino da prática baseada em evidências na formação dos enfermeiros?

Deste ponto, observa-se escassez de estudos que reflitam sobre esta temática pela perspectiva do trabalho do docente. Tem se sugerido o empreendimento de pesquisas que abordem as dificuldades do corpo docente no ensino desta prática, sobre as políticas existentes para apoio deste processo, em especial no âmbito na América Latina e Caribe, onde os estudos sobre o tema são incipientes ${ }^{4,5}$.

Acrescenta-se que nas universidades públicas brasileiras, durante a atuação dos docentes, em alguma fase de suas carreiras, poderão ser-lhes atribuídas atividades de gestão universitária. Análises sobre a atuação na gestão universitária, entre enfermeiros docentes, são recentes $^{6}$. Ao se pensar no ensino da prática baseada em evidência e na importância de sua difusão durante a formação de enfermeiros, abordar essa questão pela perspectiva de enfermeiros gestores universitários torna-se crucial. Isso se deve ao fato de que são lideranças instituídas, capazes de induzir mudanças que elevem a cooperação entre elementos importantes para o ensino dessa prática ${ }^{4,5}$.

A teoria da Difusão de Inovações tem sido empreendida para o alcance do engajamento de lideranças e indução da cultura organizacional para que seja favorável à implementação da prática baseada em evidência, especialmente por explicitar como as pessoas aderem às inovações. Distribuída em uma curva gaussiana, a difusão de inovações é adequada quando inovadores (out of the box thinkers), aqueles capazes de reconhecer o potencial de inovação precocemente - compõem 2,5\% do contingente 7,8 .

Em sequência, apresentam-se os líderes de opinião, lideranças caracterizadas como pessoas que encorajariam outros a adotarem a inovação (stakeholders), compondo 13,5\%. Pessoas que seguem as orientações dessas lideranças são 34\% do contingente. Por conseguinte, 
a curva compreende uma maioria tardia de seguidores (também de 34\%). Por sua vez, um contingente de $16 \%$ de pessoas tem comportamento tradicional, pois apresentam maiores dificuldades para adoção de mudanças, adotando-as apenas se estas apresentarem resultados práticos visíveis ${ }^{7,8}$.

Conforme esse referencial teórico, a difusão da inovação respeita quatro fases: compreender as variáveis do sistema social, quanto à necessidade de mudança e de incorporação da inovação (Fase 1); persuadir quanto às vantagens relativas a serem alcançadas pela adoção da inovação (Fase 2); decidir quanto à adoção ou rejeição da inovação (Fase 3); confirmar e difundir os resultados obtidos considerando a reorientação do sistema pela incorporação da inovação (Fase 4) (7, $^{7}$.

Tendo em vista a necessidade da difusão do ensino da prática baseada em evidências e a posição dos enfermeiros gestores como stakeholders potenciais, o presente estudo tem como objetivo compreender o ensino da prática baseada em evidências na perspectiva de enfermeiros gestores universitários.

\section{MÉTODO}

Trata-se de um estudo por métodos mistos, promovendo o entendimento sobre fenômenos ao vincular métodos quantitativos e qualitativos, por considerar que a compreensão do fenômeno não pode ser alcançada somente por uma dessas abordagens. Nos métodos mistos é importante destacar a adoção de um referencial teórico guia a execução do estudo e a análise dos dados, podendo estar implícita, bem como descrever a forma como foi realizada a conexão dos achados ${ }^{9}$.

Para o presente estudo, adotou-se a estratégia exploratória sequencial por meio da conexão dos achados, iniciada pela abordagem qualitativa que embasou a fase quantitativa. Inicia-se de uma compreensão qualitativa e contextualizada sobre os aspectos que requerem mudanças no sistema social para o ensino da prática baseada em evidências. Sequencialmente, uma análise quantitativa que investigou se existia concordância entre os participantes quanto suas percepções sobre as variáveis que compunham o sistema social frente às necessidades de mudança. $O$ referencial teórico do estudo apoia-se na concepção da prática baseada em evidência na Enfermagem ${ }^{2}$ e na Teoria de Difusão de inovações ${ }^{8}$, com ênfase na fase 1: conhecimento das variáveis do sistema social frente a necessidade de mudança.

O cenário de estudo foi uma instituição de ensino superior pública no estado de Minas Gerais, Brasil, que oferta, desde 1988, a graduação em Enfermagem. No ano de 2020, apresenta formação em Enfermagem nos níveis: técnico, bacharelado, pós-graduação lato sensu (modalidade residência) e stricto sensu (mestrado e doutorado). Vincula-se ao Sistema Único de Saúde do Brasil para composição dos cenários de integração ensino-serviço.

A população do estudo foi composta por uma amostra intencional de enfermeiros atuantes em atividades da gestão universitária, tidos aqui como potenciais lideranças para difusão do processo de inovação: o ensino da prática baseada em evidências.

A identificação dos enfermeiros gestores universitários se deu por levantamento prévio, por meio de busca ativa, em sítio eletrônico da instituição. Foram identificados aqueles que ocupavam os seguintes cargos na autarquia institucional. Foram incluídos aqueles com mandato em exercício do cargo de gestão e que o ocupavam de forma titular, e excluídos aqueles em afastamento ou férias no momento da coleta.

A coleta de dados ocorreu entre agosto e dezembro de 2017, pela condução de entrevistas individuais semiestruturadas em profundidade, que foram agendadas por contato telefônico prévio e ocorreram em local apropriado na instituição, definido pelo enfermeiro gestor. Foram realizadas por pesquisador treinado, com duração de aproximadamente 60 minutos.

0 roteiro das entrevistas foi composto por caracterização sociodemográfica e matriz sobre estratégias para o ensino da prática baseada em evidências na formação de enfermeiros. 
A organização dos componentes dessa matriz pautou-se numa revisão integrativa detalhada em publicação recente ${ }^{5}$.

A matriz é composta por três dimensões: estratégias mediadoras do processo ensinoaprendizagem (cinco itens); estratégias propiciadas pelas tecnologias de informação e comunicação (três itens); e estratégias para reorientação da matriz educacional (cinco itens). Cada item apresenta-se por uma sentença com verbo no imperativo sobre uma iniciativa viabilizadora ao ensino da prática baseada em evidências para formação de enfermeiros. Durante as entrevistas, realizou-se a leitura de cada item da matriz, sendo a seguir solicitado que o enfermeiro gestor universitário comentasse de forma livre como percebia as estratégias contidas nos itens frente ao contexto da instituição. Foram registrados no instrumento de coleta os comentários dos participantes.

A produção de dados foi obtida pela perspectiva qualitativa frente a concepção hermenêutica-dialética, que tem valorizado o poder de reflexão dos participantes do estudo. Dessa maneira, a partir de uma relação dialética entre a explicação e a compreensão de um fenômeno sob investigação, possibilitada pela reflexão a partir da interação junto a matriz sobre estratégias para o ensino da prática baseada em evidências, orientadoras das entrevistas individuais em profundidade. Com isso, por meio de um processo reflexivo e contextualizado, buscou-se a compreensão do fenômeno estudado. Todas as práticas discursivas têm gerado, potencialmente, possibilidades abertas de composição hermenêutico-dialética para a produção de dados de pesquisas, embora os participantes estejam condicionados a contextos e compromissos ${ }^{10}$.

Foi realizada a leitura conjunta pelos pesquisadores dos comentários dos participantes, no sentido de se compreender as variáveis do sistema social, por uma análise qualitativa, quanto à necessidade de mudanças para o ensino da prática baseada em evidências - ênfase na fase 1 da Teoria de Rogers ${ }^{8}$. A análise qualitativa das entrevistas consistiu em um registro textual único conforme a estrutura de análise proposta por Minayo ${ }^{10}$. Buscou-se identificar, além das estruturas semânticas, as interações entre elas e o contexto, incluindo as estruturas sociológicas de produção da mensagem.

Foram transcritos os registros textuais dos comentários das lideranças quanto a matriz de estratégias, sendo destacado como o conjunto de enfermeiros gestores universitários que percebem ser o ensino da prática baseada em evidências no contexto investigado. E apresentada uma síntese por meio de um relato descritivo, apoiando a compreensão dos aspectos que requerem mudança no sistema social.

Para analisar a existência de concordâncias entre os enfermeiros gestores sobre os aspectos do contexto local, sistema social, sobre as necessidades de mudança quanto ao ensino da prática baseada em evidências, foi organizada a transformação dos dados qualitativos em quantitativos, conforme proposta de Camargo et al ${ }^{11}$, assim como, elaborou-se uma planilha, preenchida por consenso entre os pesquisadores deste estudo após a leitura conjunta das entrevistas.

Cada avaliação individual dos itens da matriz de estratégias, realizada pelos enfermeiros gestores universitários, recebia pontuações frente aos aspectos subjetivos das respostas. $\mathrm{Na}$ existência de respostas favoráveis aos itens, independente da frequência de aparição, as respostas recebiam pontuação um (1), e na ausência de referências ou posicionamentos negativos quanto a quaisquer itens avaliados, recebiam pontuação zero (0). Foi empregada uma proporção de concordância para cada item, com base no cálculo [( $\mathrm{n}$ ㅇ de respostas favoráveis $/ \mathrm{n}^{\circ}$ total de respostas) x100]. Foram analisadas as médias das proporções de concordância para cada uma das três dimensões e para a matriz como um todo. A resposta foi considerada favorável à percepção do participante quando a estratégia mencionada seria uma prática já existente na universidade investigada.

Foi calculado o alfa de Cronbach $(\alpha=0,8)$ para estimar a confiabilidade entre os examinadores, de forma a denotar se os pesquisadores perceberam o fenômeno de forma 
similar. 0 procedimento analítico partiu da organização de um banco de dados, através do software Statistical Package for the Social Sciences (SPSS) versão 21.0.

Quanto ao aspecto ético, foi respeitada a resolução CNS 466/2012 ${ }^{12}$, sendo esta pesquisa aprovada pelo Comitê de Ética em Pesquisa da Universidade Federal do Triângulo Mineiro, em 09 de junho de 2017, sob o parecer no 2.110 .325 e o Certificado de Apresentação para Apreciação Ética $\mathrm{n}^{\mathrm{0}}$ 63571517.5.0000.5154. Os dados foram coletados após assinatura do Termo de Consentimento Livre Esclarecido pelos participantes.

\section{RESULTADOS}

Em termos daqueles sujeitos ao estudo obteve-se: coordenação de curso técnico ( $\mathrm{n}=1)$, coordenação do curso de graduação e de seus departamentos ( $\mathrm{n}=3)$, coordenação de programas de pós-graduação lato e stricto sensu $(\mathrm{n}=5)$, atuantes em cargos de gestão em pró-reitorias $(n=4)$, totalizando 13 enfermeiros gestores. A amostra final compôs-se pela participação de 10 enfermeiros gestores universitários, $77 \%$ da população pretendida.

Os pesquisados caracterizavam-se por terem média de idade de 51 anos, desvio padrão de $\pm 7,9$ anos. Apenas um respondente era do sexo masculino, oito participantes autodeclararam cor da pele branca, cinco em união estável. Todos apresentavam como vínculo principal a docência universitária, com tempo médio de atuação no ensino superior de 13,9 anos e mediana de 14 anos. Apresentavam elevada titulação, em que dois deles possuiam nível de formação pós-doutoral enquanto os demais possuíam doutorado. Na atuação na gestão universitária, cinco deles atuavam em coordenação de pós-graduação, três em coordenação da graduação e dois na autarquia da universidade como pró-reitores.

De acordo com a matriz, frente a dimensão das estratégias mediadoras do processo ensino-aprendizagem, os participantes concordaram em $80 \%$ que ocorre o desenvolvimento de iniciativas como discutir casos clínicos e gerenciais e realizar leitura, análise e revisão crítica de artigos científicos.

Já em relação à elaboração de roteiros sobre a integração de evidências na prática, 50\% concordaram que essa atividade tem sido desenvolvida (Tabela 1). A média da porcentagem de concordância para a dimensão foi de $70 \%$, sendo a maior quanto ao desenvolvimento das iniciativas no cenário investigado.

Tabela 1. Concordância dos enfermeiros gestores universitários sobre o ensino da prática baseada em evidências conforme estratégias mediadoras do processo ensino-aprendizagem. Uberaba, MG, Brasil, 2017.

\section{Estratégias mediadoras do processo ensino-aprendizagem ${ }^{\mathrm{a}}$}

$(\%)^{\mathbf{b}}$

- Discutir durante os estágios casos clínicos e gerenciais que possibilitem a identificação de uma questão clínica e a busca de evidências para respondê-la

- Realizar atividades que contenham a leitura, análise e revisão crítica de artigos científicos com diferentes delineamentos metodológicos

- Orientar o desenvolvimento de habilidades sobre a escrita de artigos científicos e a apresentação oral de resultados de pesquisa

- Elaborar projetos colaborativos de pesquisa e intervenção entre estudantes e enfermeiros do cenário de prática

- Elaborar roteiros para orientar os estudantes sobre a integração de evidências científicas na prática assistencial

$80 \%$

$80 \%$

$70 \%$

$70 \%$

${ }^{a}$ Sentenças extraídas de Camargo e cols ${ }^{5}$

${ }^{\mathrm{b}}$ Concordância $=\left[\left(\mathrm{n}^{\circ}\right.\right.$ de respostas favoráveis $/ \mathrm{n}^{\circ}$ total de respostas $\left.) \times 100\right]$.

Quanto às estratégias propiciadas pelas Tecnologias de Informação e Comunicação, todos os participantes conceberam que não há organização de espaços virtuais colaborativos entre as escolas de enfermagem e/ou universidades. Concordaram, em proporção de 70\%, que há realização de iniciativas relacionadas à facilitação do acesso para pesquisas aos estudantes de enfermagem no contexto investigado (Tabela 2). A média da porcentagem de concordância para a dimensão foi de $43,4 \%$. 
Tabela 2. Concordância dos enfermeiros gestores universitários sobre o ensino da prática baseada em evidências conforme estratégias propiciadas pelas Tecnologias de Informação e Comunicação. Uberaba, MG, Brasil, 2017.

Estratégias propiciadas pelas Tecnologias de Informação e Comunicação ${ }^{a}$

$(\%)^{\mathbf{b}}$

- Facilitar o acesso às pesquisas dos estudantes de enfermagem, por meio, por exemplo, de recursos informacionais e bases de dados

- Divulgar grupos de pesquisas e redes colaborativas de produção de conhecimento e inovações aos $70 \%$ estudantes de enfermagem

- Organizar espaços virtuais colaborativos entre as escolas de enfermagem e/ou universidades para que possam difundir inovações, ampliar a utilização da prática baseada em evidências e discutir as dificuldades para o seu ensino

a Sentenças extraídas de Camargo e cols ${ }^{5}$

${ }^{\mathrm{b}}$ Concordância $=\left[\left(\mathrm{n}^{\circ}\right.\right.$ de respostas favoráveis $/ \mathrm{n}^{\circ}$ total de respostas $\left.) \times 100\right]$.

As estratégias para orientação da matriz educacional foram tidas como as de menor frequência de ocorrência no contexto investigado. Todos os respondentes apontaram para uma ausência de convergência do projeto político pedagógico ao ensino da prática baseada em evidências (Tabela 3). Nesta perspectiva, foi a dimensão que apresentou menor média de concordância quanto à ocorrência das estratégias no cenário investigado (22\%).

Tabela 3. Concordância dos enfermeiros gestores universitários sobre o ensino da prática baseada em evidências conforme estratégias para orientação da matriz educacional. Uberaba, MG, Brasil, 2017.

\begin{tabular}{lc}
\hline Estratégias para orientação da matriz educacionala & (\%) $^{\mathbf{b}}$ \\
\hline - Fortalecer as parcerias entre escolas de enfermagem/universidades e serviços de saúde & $60 \%$ \\
- Formar enfermeiros doutores para que atuem como consultores no desenvolvimento de & \\
pesquisas, avaliação e incorporação de seus resultados na prática assistencial, em hospitais ou & $20 \%$ \\
outros serviços de saúde & $20 \%$ \\
- Integrar no currículo tópicos que abordem as etapas para implementação da prática baseada em & \\
evidências & $10 \%$ \\
- Ofertar cursos ou disciplinas complementares que deem aos estudantes a oportunidade de & \\
experimentar a geração e incorporação de resultados de pesquisa na prática & $0 \%$ \\
\hline - Orientar a matriz pedagógica para a Prática Avançada em Enfermagem, a fim de desenvolver o & \\
julgamento crítico, e o estímulo a inovações para a solução de problemas, por meio de um modelo \\
colaborativo, de ciências translacionais e sensível aos aspectos socioculturais \\
Sentenças extraídas de Camargo e cols 5
\end{tabular}

A concordância média total para as três dimensões foi de 45,4\%. Quanto à confiabilidade, o grau com que diferentes examinadores percebem o ensino da prática baseada em evidências apresentou-se consistente, $\operatorname{com} \alpha=0,823$.

A respeito da análise qualitativa do sistema social, o grupo de enfermeiros gestores percebem que a ampla difusão das tecnologias da informação e comunicação e o contexto econômico exige uma postura inovadora e empreendedora da enfermagem frente a este contexto. Eles reconhecem que a instituição oferece infraestrutura tecnológica necessária para que a PBE seja implementada sendo os espaços de colaboração virtual interinstitucionais inexistentes. Apontam também a necessidade de mudanças nos projetos políticos pedagógicos dos cursos, formação docente e percebem que existem iniciativas da instituição e dos próprios docentes na implementação da PBE, conforme detalhado no Quadro 1. 
Quadro 1. Perspectivas dos enfermeiros gestores universitários sobre ensino da prática baseada em evidências. Uberaba, MG, Brasil, 2017.

Participantes compreendem que as estratégias didáticas precisam ser inovadas, incluindo métodos correspondentes ao contexto contemporâneo, frente a adventos como internet, rápido acesso a artigos e bases de dados (até mesmo via celular) e especificidades comportamentais da nova geração de estudantes. Discutiram quais desafios atuais requerem que o enfermeiro seja capaz de inovar e empreender, controlar custos e ter prática ambientalmente sustentável. Então, o consenso é de que é necessário reinventar a sala de aula e as disciplinas, repensar estratégias de ensino. Informam que a universidade conta com boa estrutura de informática para o ensino, disponibilizando laboratórios, mas a instituição ainda não pensou espaços virtuais colaborativos para a prática baseada em evidências. Entretanto, a instituição disponibiliza acesso amplo, para toda comunidade acadêmica, a bases importantes na geração de evidências, como a Cochrane, além de contar com serviço de bibliotecárias especializadas para orientar a busca e utilização de bases de dados científicas. Relatam que aproximações do tipo são iniciativas induzidas já na graduação, em disciplinas como informática na enfermagem ou pesquisa em saúde. Apontam ser obrigatório o trabalho de conclusão de curso para obtenção de bacharelado em Enfermagem, sendo ele resultante de uma pesquisa, mas que esse acompanhamento pode ser aprimorado a fim de também ser um espaço para o ensino da prática baseada em evidências. Com isso, compreendem que a pesquisa tem sido um aspecto induzido pela instituição desde a graduação, incluindo a participação em projetos de pesquisa e disponibilização de fomentos como bolsas de iniciação científica. Contudo, refletem que é preciso ocorrer melhores direcionamentos junto aos docentes e pesquisadores para que também haja oportunidade de experiências com a prática baseada em evidências. Mencionam existir um afastamento dos grupos de pesquisa do espaço da sala de aula. Apesar de docentes serem líderes de grupos de pesquisa na instituição, não observam uma ação consistente, não há agregação direta desses grupos com disciplinas ministradas. Enfatizam que no Brasil há um sítio eletrônico de domínio público mantido pelo Conselho Nacional de Desenvolvimento Científico e Tecnológico (CNPq) no qual podem ser identificados pesquisadores e grupos de pesquisa, mas esse acesso para buscas precisa ser melhor difundido entre os estudantes. Percebem ser necessário e urgente formar enfermeiros como consultores e mentores para a (des)incorporação de evidências e tecnologias nos serviços de saúde. Relatam que as pós-graduações da instituição têm se voltado para formar docentes, pesquisadores ou especialistas, sendo crucial incrementar essa discussão na pós-graduação. Mencionam que existem iniciativas com essa convergência que ficam a cargo de poucos docentes e de forma isolada. Sobretudo, percebem a necessidade em adequar o projeto político pedagógico para a formação de enfermeiros nos diferentes níveis, considerando o ensino da prática baseada em evidências. Relatam que, na maioria das vezes, o docente traz consigo uma perspectiva do ensino, que se apoia na compartimentalização do conhecimento e na reprodução de conteúdo específico. Enfatizam a necessidade de se revisar também a formação docente. Para tanto, seria crucial mobilizar esses docentes para a prática baseada em evidências, capacitando-os melhor no tema, colocando isso como pauta de discussão junto aos departamentos didáticos, sendo os passos para se alcançar a revisão do projeto político pedagógico, para a construção de uma nova matriz de ensino. Apesar de conceberem a indução desse processo como atribuição da autarquia, destacam que deve ser um processo de engajamento do coletivo dos docentes. Outro aspecto em comum mencionado foi o fato de que participar da entrevista acabou por motivá-los a pensar sobre o tema e ampliou seu interesse pelos itens da matriz, iniciativas ali propostas.

\section{DISCUSSÃo}

Os resultados apresentam a perspectiva de um grupo de enfermeiros gestores universitários imersos no contexto de uma instituição. Eles têm percebido que o ensino da prática baseada em evidências é realizado em iniciativas pontuais, recorrendo nas estratégias mediadoras do processo ensino-aprendizagem, porém sem orientação institucional do projeto político pedagógico. Sobretudo, apontaram que é preciso engajamento junto ao corpo docente para superar essa realidade e implementar inovações. Pode ser observado que o cenário tem iniciativas voltadas para a produção e consumo de pesquisas, desde a graduação. Entretanto, existem lacunas, que incluem: estratégias de ensino, conteúdos, disciplinas, integração com cenários de prática, formação docente, matriz pedagógica e política institucional apoiadora.

Em concordância com os resultados do presente estudo, uma pesquisa documental identificou que há um movimento de mudança na formação do enfermeiro brasileiro; mas para vencer os desafios da mudança curricular e implementar o novo na formação do enfermeiro é necessário diminuir as resistências às mudanças, ao se propor reflexões sobre a docência, buscar estratégias que levem à diminuição do distanciamento dos serviços de saúde, com o reforço à clássica dicotomia entre o pensar e o fazer, e superar a fragmentação e o tecnicismo, 
presentes em boa parte da prática dos docentes ${ }^{13}$. Discute-se que os currículos de Enfermagem estão pautados no modelo médico hospitalocêntrico para a formação, sendo necessário investir para uma formação à guisa do pensamento crítico nos diferentes contextos de atenção ${ }^{13,14}$.

Sobremaneira, a formação em Enfermagem deve vislumbrar mais que a preparação técnico-científica do profissional. Deve ter como meta educar os sujeitos sociais políticos capazes de atuar responsavelmente e transformar a realidade, principalmente na redução de desigualdades como a falta de acesso ao sistema de saúde por boa parte da população ${ }^{14}$.

A prática baseada em evidências é um elemento crucial para a formação orientada pelo pensamento crítico e fundamental nas complexas configurações de cuidados de saúde atuais. Nesse sentido, tem sido crescente o investimento em modelos e frameworks que possam orientar o ensino da prática baseada em evidências ${ }^{15-17}$. Todavia, somente quando o corpo acadêmico (pesquisadores e docentes) está bem preparado, com habilidades e atitudes positivas, ele incorpora de fato a prática baseada em evidências em seu ensino e apoia o aprendizado dos alunos ${ }^{15,16 .}$

A produção científica sobre planos de implementação de medidas nos currículos para capacitar estudantes de enfermagem a aplicarem a prática baseada em evidências ainda tem lacunas ${ }^{15-17}$. Críticas apontam que simplesmente ensinar sobre pesquisa não é suficiente para garantir que a aprendizagem se traduza em prática. Embora mais enfermeiros do que nunca sejam considerados academicamente qualificados e conhecedores da teoria da pesquisa, isso não garante a transferência dessas habilidades e conhecimentos para a prática ${ }^{17}$.

Na Colômbia, docentes e pesquisadores de enfermagem da Universidade de Medellín identificaram diversas barreiras à prática baseada em evidências como: falta de incentivos para envolvimento dos enfermeiros em pesquisa; disponibilidade limitada de evidências de enfermagem; e falta de comunicação entre os ambientes de prática e academia. Parcerias para pesquisa entre universidades e instituições clínicas foram denotadas como principal facilitador ${ }^{18}$.

Outro aspecto, a gestão universitária realizada por enfermeiros docentes remete a um conjunto de ações individuais e coletivas, pertencentes a um espaço social em que se articulam grupos específicos, singulares e complexos. Em muito, o produto de todos os esforços despendidos pelos enfermeiros gestores visa o alcance de uma formação universitária de qualidade para a excelência de futuros enfermeiros ${ }^{19}$.

Dessa perspectiva, inovações, como a prática baseada em evidências, desempenham papel importante nas organizações - mesmo nas universidades, quando indicam as oportunidades internas que beneficiam seu desenvolvimento de forma sustentável, e geram benefícios ou respostas que possam atender às necessidades do contexto específico ${ }^{20}$.

Com isso, o presente estudo permitiu, no contexto da difusão da prática baseada em evidências enquanto inovação para o ensino, uma análise para compreender as condições que permeiam sua implementação. Os enfermeiros gestores universitários, como lideranças instituídas, têm um papel a desempenhar na identificação de necessidades do contexto, para, com isso, apoiar resoluções e ampliar cooperações para a implementação da inovação. Ainda, infere-se que o ato de participar de estudos sobre a temática pode induzir aos enfermeiros gestores universitários a refletirem sobre o engajamento favorável à implementação da prática baseada em evidência.

Em consonância com o disposto para a fase 1 da Teoria de Rogers ${ }^{8}$, as interações hermenêutico dialéticas empreendidas permitiram reconhecimento dos aspectos contextuais que influenciam o sistema social da universidade quanto à adoção do ensino da prática baseada em evidências na formação de enfermeiros. Variáveis foram identificadas no estudo como propulsoras para o processo de mudança rumo ao processo de ensino-aprendizagem com base nas práticas baseadas em evidências, em especial quanto ao fortalecimento das estratégias para orientação da matriz educacional e das estratégias propiciadas pelas Tecnologias de Informação e Comunicação. 


\section{CONCLUSÃo}

A prática baseada em evidências tem sido apresentada como desafio à atuação de enfermeiros em âmbito mundial. Os resultados incrementam a produção de conhecimento sobre a temática, ainda insuficiente para a América Latina e Caribe, apresentando a perspectiva de enfermeiros gestores universitários de uma instituição pública de ensino superior do Brasil.

A abordagem hermenêutico-dialética empreendida, orientada pela matriz sobre estratégias para o ensino da prática baseada em evidências, permitiu o reconhecimento de aspectos que condicionam o contexto dessa prática na instituição investigada. As respostas apresentaram concordâncias consistentes entre os entrevistados $(\alpha=0,823)$.

Foi mais limitada na instituição a existência de iniciativas que abordem o projeto político educacional e a organização de espaços virtuais colaborativos para difundir a utilização da prática baseada em evidências, sendo seu ensino usualmente empreendido no contexto investigado por iniciativas pontuais de docentes, durante estágios clínicos que possibilitem a identificação de uma questão clínica e a busca de evidências para respondê-la, ou ainda, em atividades que contemplem a revisão crítica de artigos científicos.

Como limitações desta pesquisa, a metodologia utilizada impede generalizações ao se considerar a etapa quantitativa do estudo. Entretanto, os entrevistados compuseram quase a totalidade do grupo de interesse, e as interações durante as entrevistas proporcionaram acesso a sentidos contextuais, permitindo a observância de aspectos que condicionam a implementação da prática baseada em evidências no ensino, os quais, mediante a discussão dos resultados, potencialmente correspondem a cenários similares. Todavia, a própria matriz utilizada para orientar as entrevistas no presente estudo, apresenta-se como uma ferramenta para orientar diagnósticos junto a enfermeiros gestores universitários de outras localidades.

Quanto às implicações para a prática, no âmbito individual, para os participantes da pesquisa, as reflexões que emergiram acabaram por motivá-los a pensar sobre o tema e ampliar o interesse sobre as iniciativas propostas na matriz, repercutindo positivamente. Além disso, os resultados podem orientar a formulação de políticas locais e a reorientação da matriz educacional na formação de enfermeiros.

Novas pesquisas podem ser empreendidas junto enfermeiros gestores de outras instituições universitárias, pela replicação deste método, a fim de apoiar diagnósticos locais, por conseguinte, a implementação da Prática Baseada em Evidências na formação de enfermeiros.

\section{REFERÊNCIAS}

1. Boude OF. New knowledge required for nursing practice in a dynamic and changing society. Aquichan [Internet]. 2019 [citado em 2 fev 2019]; 19(1):e1911. Disponível em: http://aquichan.unisabana.edu.co/index.php/aquichan/article/view/10250

2. Melnyk BM, Gallagher-Ford L, Fineout-Overholt E. The establishment of evidence based practice competencies for practicing registered nurses and advanced practice nurses in realworld clinical settings: proficiencies to improve healthcare quality, reliability, patient outcomes and costs. Worldviews Evid Based Nurs. [Internet]. 2014 [citado em 10 fev 2019]; 11(1):5-15. Disponível em: https://sigmapubs.onlinelibrary.wiley.com/doi/epdf/10.1111/wvn.12021

3. Camargo FC, Iwamoto HH, Galvão CM, Pereira GA, Andrade RB, Masson GC. Competences and barriers for the evidence-based practice in nursing: an integrative review. Rev Bras Enferm. [Internet]. 2018 [citado em 13 dez 2018]; 71(4):2030-8. Disponível em: http://www.scielo.br/pdf/reben/v71n4/0034-7167-reben-71-04-2030.pdf

4. Camargo FC, Antunes M, Andrade RB, Monteiro DAT, Iwamoto HE, Pereira GA. Docentes pesquisadores, investigações de enfermagem e estratégias para translação do conhecimento em hospital universitário. Ciênc Cuid Saúde [Internet] 2018 [citado em 12 jan 2019]; 17(2):18. Disponível

em: http://periodicos.uem.br/ojs/index.php/CiencCuidSaude/article/view/41637 
5. Camargo FC, Iwamoto HH, Pereira GA, Souza RM, Garcia LAA, Monteiro DAT, et al. Estratégias para o ensino da prática baseada em evidências na formação de enfermeiros: revisão integrativa. REFACS [Internet]. 2018 [citado em 10 jan 2019]; 6(Supl1):363-74. Disponível em: http://seer.uftm.edu.br/revistaeletronica/index.php/refacs/article/view/2288

6. Cunha KS, Andrade SR, Erdmann AL. University management nurse: a grounded theory. Rev Latinoam Enferm. [Internet]. 2018 [citado em 8 jan 2019]; 26:e2980. Disponível em: http://www.scielo.br/pdf/rlae/v26/0104-1169-rlae-26-e2980.pdf

7. Almeida JPL, Farias JS, Carvalho HS. Drivers da adoção de tecnologias em serviços de saúde. BBR Braz Bus Rev. [Internet] 2017 [citado em 14 fev 2019]; 14(3):336-51. Disponível em: http://www.scielo.br/pdf/bbr/v14n3/pt_1808-2386-bbr-14-03-0336.pdf

8. Rogers EM. Diffusion of innovations. Nova York: Free Press; 2003. 576p.

9. Oliveira JLC, Magalhães AMM, Matsuda LM. Métodos mistos na pesquisa em enfermagem: possibilidades de aplicação à luz de Creswell. Texto \& Contexto Enferm. [Internet]. 2018 [citado em 9 fev 2019]; 27(2):e0560017. Disponível em: http://www.scielo.br/pdf/tce/v27n2/01040707-tce-27-02-e0560017.pdf

10. Minayo MCS. O desafio do conhecimento. Pesquisa qualitativa em saúde. São Paulo: Hucitec; 2010.

11. Camargo FC, Iwamoto HH, Monteiro DAT, Lorena LT, Pereira GA. Avaliação de intervenção para difusão da enfermagem baseada em evidências em hospital de ensino. Rev Gaúcha Enferm. [Internet]. 2016 [citado em 2 mar 2019]; 37(Esp):e68962. Disponível em: http://www.scielo.br/pdf/rgenf/v37nspe/0102-6933-rgenf-1983-14472016esp68962.pdf

12. Conselho Nacional de Saúde (Brasil). Resolução n. $\stackrel{0}{466 . ~ D i r e t r i z e s ~ e ~ n o r m a s ~}$ regulamentadoras de pesquisa envolvendo seres humanos [Internet]. Brasília, DF: Conselho Nacional de Saúde; 2012 [citado em 15 fev 2019]. Disponível em: http://bvsms.saude.gov.br/bvs/saudelegis/cns/201 3/res0466_12_12_2012.html

13. Silveira CA, Paiva SMA. A evolução do ensino de enfermagem no Brasil: uma revisão histórica. Ciênc Cuid Saúde [Internet] 2011 [citado em 2 fev 2019]; 10(1):176-83. Disponível em: http://periodicos.uem.br/ojs/index.php/CiencCuidSaude/article/view/6967/0

14. Fonseca RMGS, Barbosa DA, Egry EY. Formation in social responsibility of nursing professionals: a Brazilian perspective. Invest Educ Enferm. [Internet]. 2018 [citado em 5 fev 2019]; 36(2):e01. Disponível em: http://www.scielo.org.co/pdf/iee/v36n2/2216-0280-iee36-02-e01.pdf

15. Malik G, McKenna L, Griffiths D. An analysis of evidence-based practice curriculum integration in Australian undergraduate nursing programs. GSTF J Nurs Health Care [Internet]. 2015 [citado em 23 jan 2019]; 3:158-64. Disponível em: http://dl6.globalstf.org/index.php/jnhc/article/view/427/376

16. Malik G, McKenna L, Griffiths D. Endeavoring to contextualize curricula within an EBP framework: a grounded theory study. West J Nursing Res. [Internet]. 2018 [citado em 9 fev 2019]; 40(12):1765-84. Disponível em: https://journals.sagepub.com/doi/pdf/10.1177/0193945917753589

17. Moore F, Tierney S. What and how... but where does the why fit in? The disconnection between practice and research evidence from the perspective of UK nurses involved in a qualitative study. Nurse Educ Pract. [Internet]. 2019 [citado em 14 fev 2019]; 34:90-6. Disponível em: https://www.sciencedirect.com/journal/nurse-education-inpractice/vol/34/suppl/C

18. Debruyn RR, Ochoa-Marin SC, Semenic, S. Barriers and facilitators to evidence-based nursing in Colombia: perspectives of nurse educators, nurse researchers and graduate students. Invest Educ Enferm. [Internet]. 2014 [citado em 3 mar 2019]; 32(1):21. Disponível em: http://www.scielo.org.co/pdf/iee/v32n1/v32n1a02.pdf

19. Candela L, Gutierrez AP, Keating S. What predicts nurse faculty members' intent to stay in the academic organization? A structural equation model of a national survey of nursing faculty. 
Nurse Educ Today [Internet]. 2015 [citado em 10 mar 2019]; 35(4):580-9. Disponível em: https://www.sciencedirect.com/science/article/pii/S0260691715000027?via\%3Dihub 20. Correia SEN, Oliveira VM, Gomez CRP. Dimensions of social innovation and the roles of organizational actor: the proposition of a framework. Rev Adm Mackenzie [Internet]. 2016 [citado em 6 mar 2019]; 17(6):102-33. Disponível em: http://www.scielo.br/pdf/ram/v17n6/1678-6971-ram-17-06-0102.pdf

Editora Associada: Vania Del Arco Paschoal

CONTRIBUIÇÕES

Fernanda Carolina Camargo participou da concepção, análise dos dados, redação e revisão. Gilberto de Araújo Pereira atuou na concepção e revisão. Luan Augusto Alves Garcia contribuiu na redação e revisão. Marija Antunes participou da coleta e análise dos dados. Adriana Oliveira de Freitas Araujo e Álvaro da Silva Santos contribuíram na revisão.

\section{Como citar este artigo (Vancouver)}

Camargo FC, Pereira GA, Garcia LAA, Antunes M, Araujo AOF, Santos ÁS. Ensino da prática baseada em evidências: perspectivas de enfermeiros gestores universitários. REFACS [Internet]. 2021 [citado em inserir dia, mês e ano de acesso]; 9(2):427-37. Disponível em: inserir link de acesso. DOI: inserir link do DOI

\section{Como citar este artigo (ABNT)}

CAMARGO, F. C.; PEREIRA, G. A.; GARCIA, L. A. A.; ANTUNES, M.; ARAUJO, A. O. F.; SANTOS, Á. S. Ensino da prática baseada em evidências: perspectivas de enfermeiros gestores universitários. REFACS, Uberaba, MG, v. 9, n. 2, p. 427-37, 2021. DOI: inserir link do DOI. Disponível em: inserir link de acesso. Acesso em: inserir dia, mês e ano de acesso.

\section{Como citar este artigo (APA)}

Camargo, F.C., Pereira, G.A., Garcia, L.A.A., Antunes, M., Araujo, A.O.F., \& Santos, Á.S. (2021). Ensino da prática baseada em evidências: perspectivas de enfermeiros gestores universitários. REFACS, 9(2), 427-37. Recuperado em inserir dia, mês e ano de acesso de inserir link de acesso. DOI: inserir link do DOI. 